\title{
Chirality of electrodeposits grown in a magnetic field
}

\author{
T. R. Ní Mhíocháin and J. M. D. Coey \\ Physics Department, Trinity College, Dublin 2, Ireland \\ (Received 18 August 2003; published 16 June 2004)
}

\begin{abstract}
Electrodeposits grown around a point cathode in a flat, horizontal electrochemical cell have fractal form. When grown in the presence of a perpendicular applied magnetic field, the deposits develop a spiral structure with chirality which reverses on switching the field direction. These structures are modeled numerically using biased variants of the diffusion limited aggregation (DLA) model. The effects of electric and magnetic fields are modeled successfully by varying the probabilities that a random walker will move in a given direction as a result of a Coulomb force and the Lorentz force-induced flow of electrolyte past the deposit surface. By contrast, a numerical model which considers only the effect of the Lorentz force on individual ions, without reference to the surface of the growing deposit, produces spiral structures with incorrect chirality. The modified DLA model is related to the differential equations for diffusion, migration, and convection. Length scales in the problem are understood by associating the step length of the random walker with the diffusion layer thickness, the lookup radius with the hydrodynamic boundary layer thickness and a point on the numerical deposit with a nucleation center for growth of a crystallite.
\end{abstract}

DOI: 10.1103/PhysRevE.69.061404

PACS number(s): 61.43.Hv, 81.15.Pq, 82.20.Wt, 81.10.Aj

\section{INTRODUCTION}

The first reports of metal structures electrodeposited with random branched geometry date back to 18th century France. Branched silver structures grown in two-dimensional cells were reported by La Condamine in 1731 [1]. Largely neglected for a century and a half, interest in these objects resurfaced in the 1980s with the development of fractal geometry by Mandelbrot [2]. Within this new fractal science, Witten and Sander [3] developed the diffusion limited aggregation (DLA) numerical model. This numerically simple, yet mathematically subtle model, based on the aggregation of "random walkers," bears striking resemblance to many structures found in nature. In particular Matsushita [4] observed its similarity to two-dimensional branched electrodeposits grown at low concentrations and low voltages. This discovery provoked newfound interest in these objects [5-7].

A typical experimental setup consists of a ring anode concentric about a small cylindrical cathode. Electrolyte consisting of a solution of a metallic salt (e.g., $\mathrm{CuSO}_{4}$ or $\mathrm{ZnSO}_{4}$ in water) is sandwiched between the electrodes by two flat perspex plates separated by $\sim 0.2 \mathrm{~mm}$.

Voltage is applied between the electrodes, and metal ions (e.g., $\mathrm{Cu}^{2+}, \mathrm{Zn}^{2+}$ ) plate from solution to the cathode. The morphologies of the resultant deposits vary [5,6] depending on applied voltage and ion concentration. In the very low voltage, low concentration limit the deposits resemble the diffusion-limited aggregates modeled by Witten and Sander. Still at low concentrations, but at higher applied voltages, deposits become more dense and grow within a clearly defined circular envelope; these are known as dense radial [7] or dense branched morphologies [8]. At high voltages and high concentrations, deposits are dendritic and even stringy. Samples of these morphologies are shown in Fig. 1. A "phase diagram" of the variation in growth with applied voltage and ion concentration is presented in Fig. 2.

The effect of an applied magnetic field $\boldsymbol{B}$ on the growth of these deposits was first investigated by Mogi et al. [9-11].
Applying a vertical magnetic field to the deposition of $\mathrm{Zn}$ and Ag in a flat horizontal cell, he found that both DLA and dendritic structures develop with a chiral form. In very high fields up to $B=8 \mathrm{~T}$, the deposits become smaller and denser. Hinds et al. [12-14] enhanced this work with a thorough investigation of $\mathrm{Cu}$ deposition in both vertical and horizontal cells, with magnetic fields applied both in and out of the plane of growth in each case. We have recently completed an extensive study of the effects of magnetic field and gravity on the growth of zinc fractal electrodeposits in both concentric and parallel electrode cells [15].

Figure 3 demonstrates the effect of applying a magnetic field perpendicular to the plane of growth of a zinc deposit in a two-dimensional horizontal cell. The chirality of the deposit reverses according to whether the field is applied vertically upwards or downwards. The uniform magnetic field
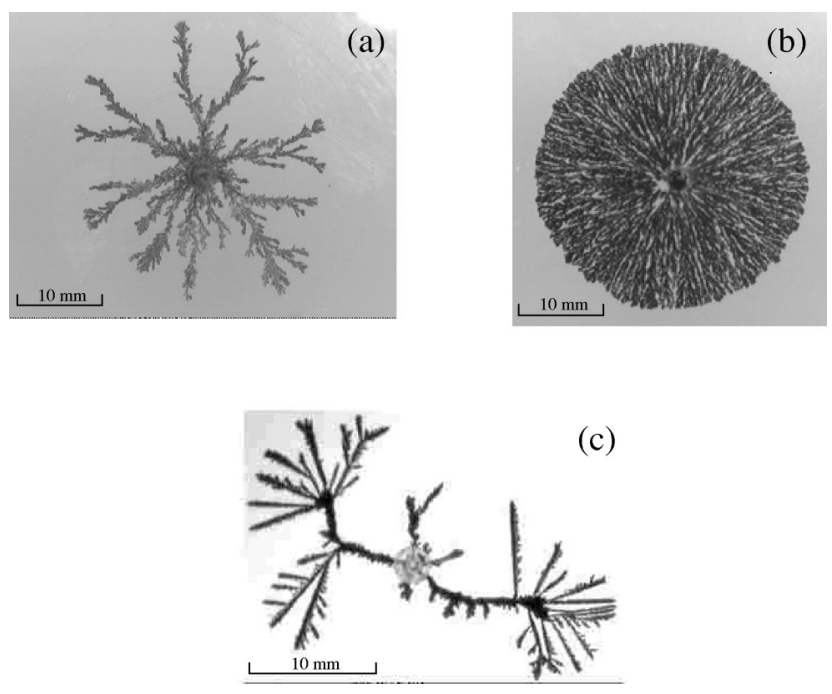

FIG. 1. Typical zinc electrodeposits grown in flat horizontal cells; (a)DLA; (b) dense radial; (c) dendritic. 


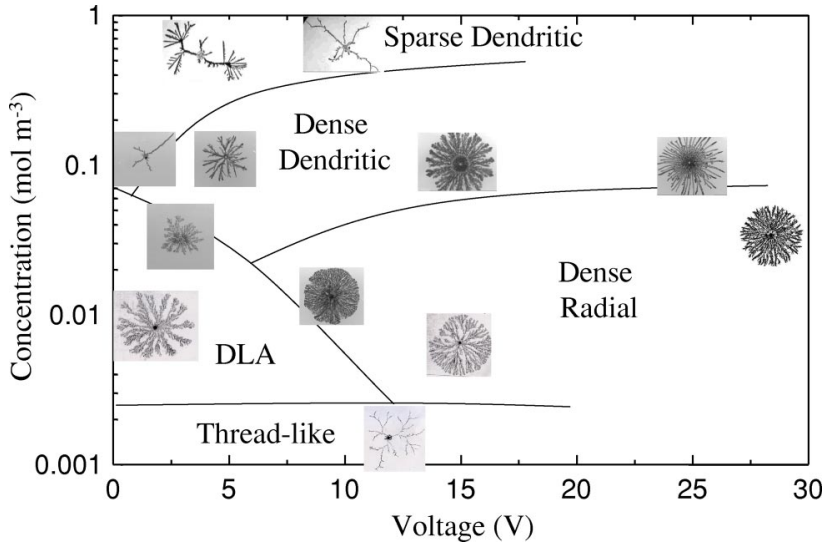

FIG. 2. Overview of the morphology of zinc electrodeposits grown in a flat horizontal cell as a function of electrolyte concentration $c_{o}$ and applied voltage $V$.

of $B=0.35 \mathrm{~T}$ used here was provided by a large Halbach cylinder permanent magnet assembly [16].

Our purpose, in this paper, is to detail a numerical approach to modeling these systems in the low concentration regime. One numerical model has been proposed by $\mathrm{Mi}$ zuseki et al. [17,18] to introduce the affect of an applied magnetic field. We outline the logic behind this model, but show that it disagrees fundamentally with the experimental result. We proceed to present an alternative magnetic field model, which produces morphologies consistent with experiment. First, we establish the relation between the DLA model and the differential equations for electrochemical mass transport.

\section{NUMERICAL SIMULATION}

The most basic version of the DLA model, as originally proposed by Witten and Sander [3], serves as a suitable model for this electrochemical system in the very low voltage, low concentration regime where diffusion of ions through a diffusion layer is the limiting factor determining growth.

At equilibrium, in two dimensions, the diffusion equation

$$
\frac{\partial c}{\partial t}=D \nabla^{2} c
$$

reduces to a Laplace equation

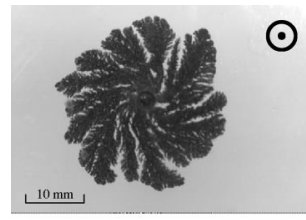

(a)

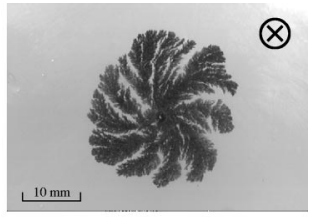

(b)
FIG. 3. Zinc electrodeposits grown from $0.1 \mathrm{M} \mathrm{ZnSO}_{4}$ at $10 \mathrm{~V}$ in a flat horizontal cell in the presence of a $0.35 \mathrm{~T}$ magnetic field applied (a) vertically upwards, and (b) vertically downwards.
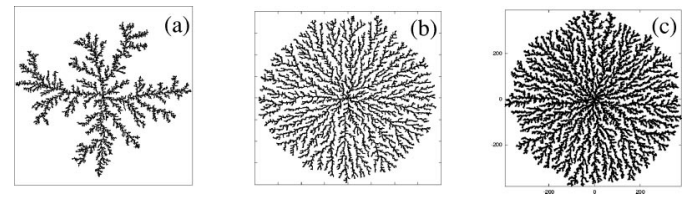

FIG. 4. (a) A basic DLA fractal; (b) Electric field model with $E_{\text {dep }}\left(d_{d e p}\right)=C / d_{d e p}, C=0.5$ and $E_{c e n}=0.05$; (c) Multiple particle simulation with a concentration represented by $f_{c}=0.2$.

$$
\nabla^{2} c=\frac{\partial^{2} c}{\partial x^{2}}+\frac{\partial^{2} c}{\partial y^{2}}=0
$$

where $c$ is the molar concentration of an ionic species in solution and $D$ is its diffusion constant. In discrete form, we can write this equation as

$$
\begin{aligned}
& \left\{\frac{\left[c\left(x_{i+1}, y_{j}\right)-c\left(x_{i}, y_{j}\right)\right]-\left[c\left(x_{i}, y_{j}\right)-c\left(x_{i-1}, y_{j}\right)\right]}{\Delta^{2}}\right\} \\
& +\left\{\frac{\left[c\left(x_{i}, y_{j+1}\right)-c\left(x_{i}, y_{j}\right)\right]-\left[c\left(x_{i}, y_{j}\right)-c\left(x_{i}, y_{j-1}\right)\right]}{\Delta^{2}}\right\}=0
\end{aligned}
$$

in order to find a solution for the concentration $c\left(x_{i}, y_{j}\right)$ at a point $\left(x_{i}, y_{j}\right)$ on a finite square grid with equal separation $\Delta x=\Delta y=\Delta$ between neighboring sites. This Laplace equation thus has discrete solution in two dimensions

$$
c\left(x_{i}, y_{j}\right)=\frac{c\left(x_{i+1}, y_{j}\right)+c\left(x_{i-1}, y_{j}\right)+\left(x_{i}, y_{j-1}\right)+c\left(x_{i}, y_{j+1}\right)}{4}
$$

relating the concentration at site $\left(x_{i}, y_{j}\right)$ on a finite grid to the concentrations at neighboring sites. This finite difference solution to the Laplace equation has, however, the same form as the probability $P\left(x_{i}, y_{j}\right)$ equation [19]

$$
P\left(x_{i}, y_{j}\right)=\frac{P\left(x_{i+1}, y_{j}\right)+P\left(x_{i-1}, y_{j}\right)+P\left(x_{i}, y_{j+1}\right)+P\left(x_{i}, y_{j-1}\right)}{4}
$$

for the movement of a random walker to a site $\left(x_{i}, y_{j}\right)$, which depends on the probabilities that it previously occupied one of the four neighboring sites. This similarity between the discrete solution of the diffusion equation and the motion of a random walker marks its usefulness in modeling diffusionlimited systems.

In the model, the electrochemical cell is represented by a two-dimensional square lattice, with the central cathode identified by an occupied site at the center of the grid. A particle (random walker) enters the grid. It moves about the grid with equal probability $1 / 4$ of moving up, down, left or right at each step in accordance with Eqs. (4) and (5). It continues to move in this way until it reaches a site adjacent to the occupied site, at which point it sticks. (If it walks off the grid, it is discarded and a new particle is introduced at a random point on the edge.) Another particle is then introduced, and moves until it sticks to an occupied neighbour and so on. In this way, patterns of the kind shown in Fig. 4(a) 
develop which resemble the low voltage, low concentration DLA electrodeposits of Fig. 1(a).

Monitoring the variation of current with applied voltage during the growth of two-dimensional electrodeposits [15], however, the diffusion-limited, low voltage plateau seen in three-dimensional growth is not reproduced. Rather a linear $I-V$ curve is observed at $\mathrm{Zn}^{2+}$ concentrations as low as $0.01 \mathrm{M}$ This indicates that growth may not be strictly diffusion limited even at low voltages and concentrations. As there is no supporting electrolyte present in the experimental cell to screen the metal ions from the applied electric field, the migration of ions through this electric field may be a dominant mass transfer mechanism. We thus introduce the effect of an applied electric field into our numerical model.

\section{A. Electric field}

Positively charged metal ions from the solution are drawn along electric field lines, perpendicular to the cathode surface according to the equation

$$
\frac{\partial c}{\partial t}=D \nabla^{2} c-\nabla(\mu c \boldsymbol{E}),
$$

where $\boldsymbol{E}$ is the electric field and $\mu$ the ion mobility. At equilibrium this reduces to

$$
D \nabla^{2} c-\mu \boldsymbol{E} \cdot \boldsymbol{\nabla} c-\mu c \boldsymbol{\nabla} \cdot \boldsymbol{E}=0 .
$$

We can also state that, in a medium with free charges,

$$
\nabla^{2} \Phi=-\nabla \cdot E=-\frac{F\left(z_{c} c_{c}-z_{a} c_{a}\right)}{\varepsilon \varepsilon_{o}}=-\rho,
$$

where $z_{c}$ and $z_{a}$ are the charges on the cations and anions, respectively, which in turn have molar concentrations $c_{c}$ and $c_{a}$, and the potential $\Phi$ satisfies $\boldsymbol{E}=-\boldsymbol{\nabla} \Phi \cdot \boldsymbol{\varepsilon}_{\mathrm{o}}$ is the vacuum permittivity, $\varepsilon$ the dielectric constant of water, and $F$ is the Faraday constant. An externally applied electric field related to a charge distribution outside the medium is, within a uniform solution, described by the Laplace equation

$$
\nabla^{2} \Phi=0 .
$$

It would appear then, that within an ideally neutral solution satisfying the electroneutrality condition

$$
\sum_{i} z_{i} c_{i}=0
$$

over all species $i$, the charge density $\rho$ would be strictly zero and the internal electric potential would also satisfy Laplace's equation. In practice, however, owing to the enormous value of $F / \varepsilon \varepsilon_{0}\left(1.4 \times 10^{14} \mathrm{Vm} / \mathrm{mol}\right.$ for a relative dielectric constant of 78), even slight deviations from electroneutrality may result in significant local electric fields. A corollary of this effect, however, is that any appreciable separation of charge would require prohibitively large electric forces, and is therefore a local or relatively minor effect. Nonetheless, although electroneutrality is commonly assumed in electrochemistry, it is incorrect to infer that Laplace's equation is necessarily satisfied by the electric potential in a given electrolyte [20].
What we assume here, however, is one of two things. Either local deviations from strict electroneutrality result in (a) fluctuations in the electric field which are randomly distributed and oriented across the cell or (b) slight gradients which are radial across the cell and directed towards the cathode resulting in a radial electric field. In the former case, the random nature of the effect of the electric field fluctuations is modeled by the random movement of the walker detailed above. In the latter case, as the net effect at local level is in the same radial direction as an externally applied electric field, it is modeled as additional to the external field and also satisfying Eq. (9).

We therefore proceed with the approximation of an externally applied electric field satisfying Laplace's equation so that Eq. (7) is simply given by

$$
\begin{gathered}
D \nabla^{2} c-\mu \boldsymbol{E} \cdot \nabla c=0 \\
\Rightarrow D\left[\frac{\partial^{2} c}{\partial x^{2}}+\frac{\partial^{2} c}{\partial y^{2}}\right]-\mu\left[E_{x} \frac{\partial c}{\partial x}+E_{y} \frac{\partial c}{\partial y}\right]=0 .
\end{gathered}
$$

Using the dimensionless variables $\bar{c}=c / c_{o}$ where $c_{o}$ is the bulk concentration; $\bar{x}=x / a$ and $\bar{y}=y / a$, where $a$ is the lattice spacing between adjacent grid points; $\bar{E}_{x}=(\mu a / D) E_{x}$ and $\bar{E}_{y}=(\mu a / D) E_{y}$; we can rewrite Eq. (12) in dimensionless form

$$
\frac{\partial^{2} \bar{c}}{\partial \bar{x}^{2}}+\frac{\partial^{2} \bar{c}}{\partial \bar{y}^{2}}=\bar{E}_{x} \frac{\partial \bar{c}}{\partial \bar{x}}+\bar{E}_{y} \frac{\partial \bar{c}}{\partial \bar{y}} .
$$

Solving this in discrete form for the concentration at grid point $\left(\bar{x}_{i}, \bar{y}_{j}\right)$ relative to the concentrations at its nearest neighbours we find that

$$
\begin{aligned}
\bar{c}\left(\bar{x}_{i}, \bar{y}_{j}\right)= & \bar{c}\left(\bar{x}_{i+1}, \bar{y}_{j}\right)\left[\frac{1}{4}-\frac{\bar{E}_{x}}{8}\right]+\bar{c}\left(\bar{x}_{i-1}, \bar{y}_{j}\right)\left[\frac{1}{4}+\frac{\bar{E}_{x}}{8}\right] \\
& +\bar{c}\left(\bar{x}_{i}, \bar{y}_{j+1}\right)\left[\frac{1}{4}-\frac{\bar{E}_{y}}{8}\right]+\bar{c}\left(\bar{x}_{i}, \bar{y}_{j-1}\right)\left[\frac{1}{4}+\frac{\bar{E}_{y}}{8}\right] .
\end{aligned}
$$

Our numerical convention defines angles $\theta$ anticlockwise from the $y$ axis so that $\bar{E}_{x}=-\bar{E} \sin \theta$ and $\bar{E}_{y}=\bar{E} \cos \theta$. We thus rewrite Eq. (14) as

$$
\begin{aligned}
\bar{c}\left(\bar{x}_{i}, \bar{y}_{j}\right)= & \bar{c}\left(\bar{x}_{i+1}, \bar{y}_{j}\right)\left[\frac{1}{4}+\frac{\bar{E}}{8} \sin \theta\right] \\
& +\bar{c}\left(\bar{x}_{i-1}, \bar{y}_{j}\right)\left[\frac{1}{4}-\frac{\bar{E}}{8} \sin \theta\right] \\
& +\bar{c}\left(\bar{x}_{i}, \bar{y}_{j+1}\right)\left[\frac{1}{4}-\frac{\bar{E}}{8} \cos \theta\right] \\
& +\bar{c}\left(\bar{x}_{i}, \bar{y}_{j-1}\right)\left[\frac{1}{4}+\frac{\bar{E}}{8} \cos \theta\right] .
\end{aligned}
$$

As with Eqs. (4) and (5), we implement this numerically in terms of the probabilities for movement between neighboring 


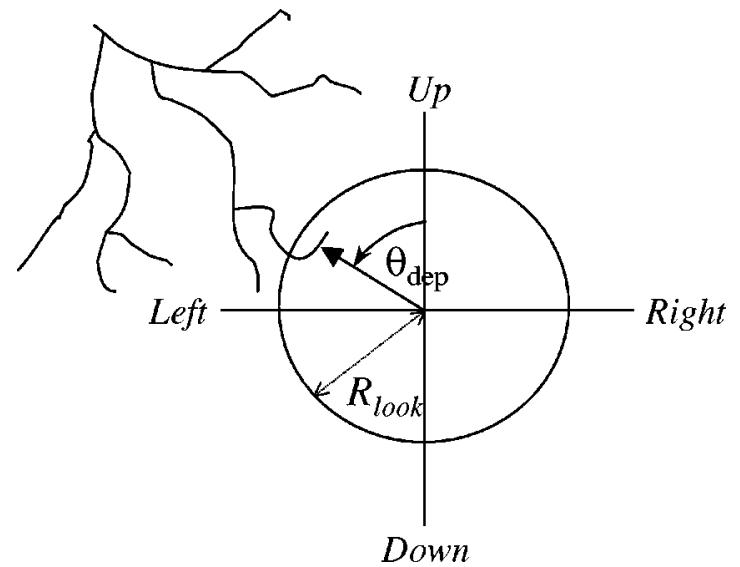

FIG. 5. Sites within a lookup radius $R_{\text {look }}$ of the particle are checked for occupation. The angle $\theta_{\text {dep }}$ (measured counterclockwise from the $y$ axis) to the nearest occupied site is then calculated.

sites. In this case, the effect of the electric field is to increase the probability that the particle will move directly towards the nearest occupied site on the surface of the deposit. This is achieved by scanning within a lookup radius $R_{\text {look }}$ about the moving particle for occupied sites [21]. In these simulations $R_{\text {look }}=10$ lattice spacings. The angle $\theta_{\text {dep }}$ to the nearest detected occupied site is calculated as shown in Fig. 5 and the probabilities for movement up, down, left or right at the next step are varied in geometric proportion to $\theta_{d e p}$, according to

$$
\begin{gathered}
\mathrm{Up}: \frac{1}{4}+E_{d e p}\left(d_{d e p}\right) \cos \theta_{d e p} \\
\text { Down: } \frac{1}{4}-E_{d e p}\left(d_{d e p}\right) \cos \theta_{d e p}, \\
\text { Left: } \frac{1}{4}+E_{d e p}\left(d_{d e p}\right) \sin \theta_{d e p}, \\
\text { Right: } \frac{1}{4}-E_{d e p}\left(d_{d e p}\right) \sin \theta_{d e p},
\end{gathered}
$$

where we use the approximation $E_{d e p}\left(d_{d e p}\right)=C / d_{d e p} . C$ is a constant and $d_{d e p}$ is the distance from the particle to the nearest occupied site. (If there is more than one "nearest" occupied site, then the probabilities of movement towards each are individually varied.)

It is, however, computationally expensive to scan the entire grid relative to the moving particle, especially when it is far from the deposit. We note, however, that the structure grows within a broadly circular envelope. Far from the deposit therefore, the electric field lines essentially emanate from the centre of the grid. We thus simplify the calculation by saying that at a distance from the furthest point of growth of the deposit, the applied voltage can be modeled simply as a drift towards center. At each step, the angle $\theta_{\text {cen }}$ (Fig. 6) from the particle to center is calculated. The probability that the particle will move towards the center of the deposit is thus increased by varying the probability that it will move up, down, left or right at the next step in geometric proportion to $\theta_{c e n}$ according to

$$
\mathrm{Up}: \frac{1}{4}+E_{c e n} \cos \theta_{c e n}
$$

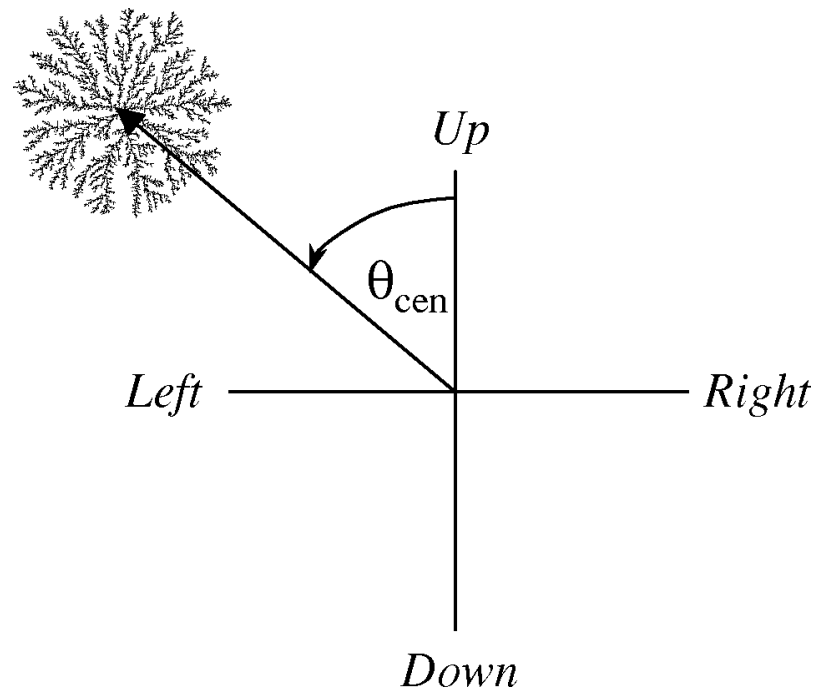

FIG. 6. At each step, the angle $\theta_{\text {cen }}$ (measured counterclockwise from the $y$ axis) between the particle and the initial central seed site is calculated.

$$
\begin{aligned}
& \text { Down: } \frac{1}{4}-E_{c e n} \cos \theta_{c e n}, \\
& \text { Left: } \frac{1}{4}+E_{c e n} \sin \theta_{c e n}, \\
& \text { Right: } \frac{1}{4}-E_{c e n} \sin \theta_{c e n},
\end{aligned}
$$

where $E_{c e n}$ is a constant indicating the strength of the applied electric field. Migration in an electric field is thus modeled through a combination of (a) a drift towards center while the moving particle is far from the deposit and (b) an increased probability of movement towards the surface when the particle is close to the deposit. (Erlebacher et al. [22] also use a separate treatment of regions close to and far from the deposit.) The resultant fractals, as shown in Fig. 4(b), are more dense and grow within a clearly defined circular envelope. This is equivalent to the dense radial electrodeposits of Fig. 1(b).

\section{B. Concentration}

The basic DLA model also assumes that only one particle is moving at a given time. This is not, however, the case even at low concentrations. We therefore introduce finite concentrations by allowing multiple particles to move and stick simultaneously. We maintain a constant concentration as the deposit grows by defining a given fraction $f_{c}$ of the sites within an annulus of thickness $N_{r e l}$ about the maximum radius of growth $r_{\max }$, which serve as starting points for random walkers. Thus as the deposit grows, the number of particles $n_{\text {movers }}$ moving in the system is constantly increasing according to

$$
n_{\text {movers }} \propto 2 \pi f_{c} N_{\text {rel }} r_{\text {max }} .
$$

No two moving particles can occupy the same site simultaneously, and as one particle sticks, a new particle is introduced within the starting annulus. 


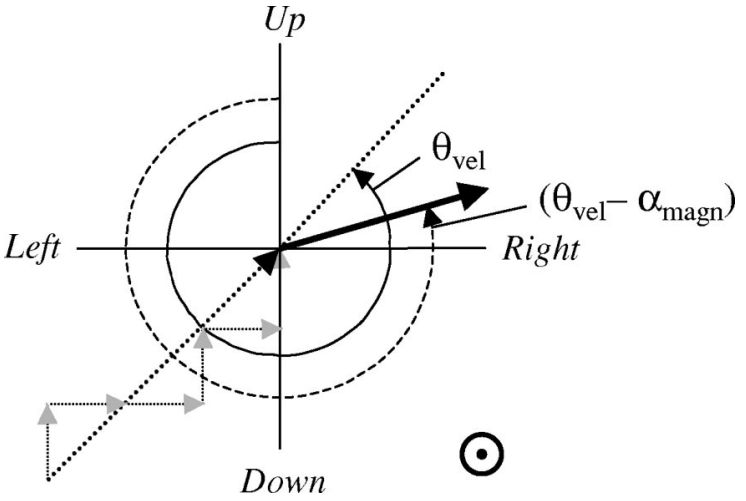

FIG. 7. The angle $\theta_{v e l}$ through which the particle has moved over the previous $N$ steps is calculated relative to the "up" direction. An applied field will tend to make it deviate from this course by an angle $\alpha_{\text {magn }}$. Here $\alpha_{\text {magn }}$ has a negative value, indicating that the magnetic field is applied vertically upwards.

The effect of this is simply to make the deposits somewhat more dense with slightly thicker branches as shown in Fig. 4(c). Multiparticle diffusion has been extensively studied by Voss [23].

\section{Magnetic field}

We begin by outlining the magnetic field model used by Mizuseki et al. [17,18] and ourselves [24] which results in chiral deposits, but which actually spiral in directions opposite to the experimental observation. We then present an alternative, more physical, magnetic field model [21] which yields results consistent with experiment.

\section{First magnetic field model}

The first magnetic field model considers that a particle with charge $q$ moving with velocity $\boldsymbol{v}$ in a magnetic field $\boldsymbol{B}$ will tend to be deflected by the Lorentz force $q \boldsymbol{v} \times \boldsymbol{B}$. It is not useful to define a velocity $\boldsymbol{v}$ for a purely random walk. In this system, however, the particle experiences a drift velocity due the applied voltage of Sec. II A, which is superimposed

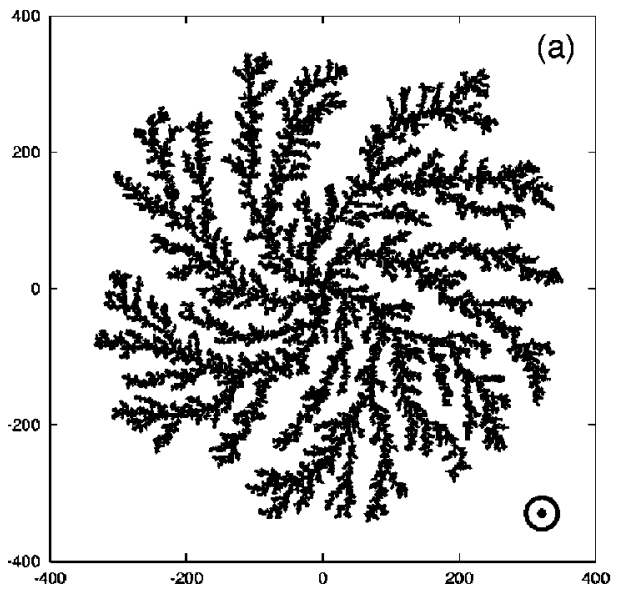

on its random movement. Numerically, the "velocity" of a particle is determined by considering its trajectory over the previous $N$ steps (here $N=6$ ). The average angle $\theta_{v e l}$ through which it has moved over $N$ steps is calculated as shown in Fig. 7. Assuming that the particle would continue along this trajectory in the absence of an applied magnetic field, we introduce a magnetic field by varying the probabilities for movement at the next step so that it will tend to be deflected by an angle $\alpha_{\text {magn }}$ from the direction defined by $\theta_{v e l}$ according to

$$
\mathrm{Up}: \frac{1}{4}+v B_{v e l} \cos \left(\theta_{v e l} \pm \alpha_{\text {magn }}\right)
$$

$$
\begin{gathered}
\text { Down: } \frac{1}{4}-v B_{v e l} \cos \left(\theta_{v e l} \pm \alpha_{\text {magn }}\right), \\
\text { Left: } \frac{1}{4}+v B_{v e l} \sin \left(\theta_{v e l} \pm \alpha_{\text {magn }}\right)
\end{gathered}
$$

$$
\text { Right: } \frac{1}{4}-v B_{v e l} \sin \left(\theta_{v e l} \pm \alpha_{\text {magn }}\right)
$$

where $B_{v e l}$ and $\alpha_{\text {magn }}$ indicate the strength of the magnetic field. The ' \pm ' of Eq. (19) is ' + ' when the magnetic field is applied vertically downwards to the horizontal cell and '-' when applied vertically upwards.

This model produces chiral results as shown in Fig. 8. The direction of the chirality is, however, opposite in direction to the experimental result of Fig. 3. We must therefore reconsider the physical content of the model.

\section{Second magnetic field model}

Consider first the example of free electrons with charge $q$ in a conducting material. They have a certain random motion due to frequent collisions with atoms of the material. An applied electric field imposes a certain drift velocity $\boldsymbol{v}_{d}$ on their movement in addition to their random motion. An applied magnetic field (in the Hall effect, for example) exerts a Lorentz force $q \boldsymbol{v}_{d} \times \boldsymbol{B}$ on each electron. The current density in the conducting material is given by $\boldsymbol{j}=n q \boldsymbol{v}_{d}$ where $n$ is the number of electrons, and the Lorentz force $\boldsymbol{j} \times \boldsymbol{B}$ on $\boldsymbol{j}$ is ef-

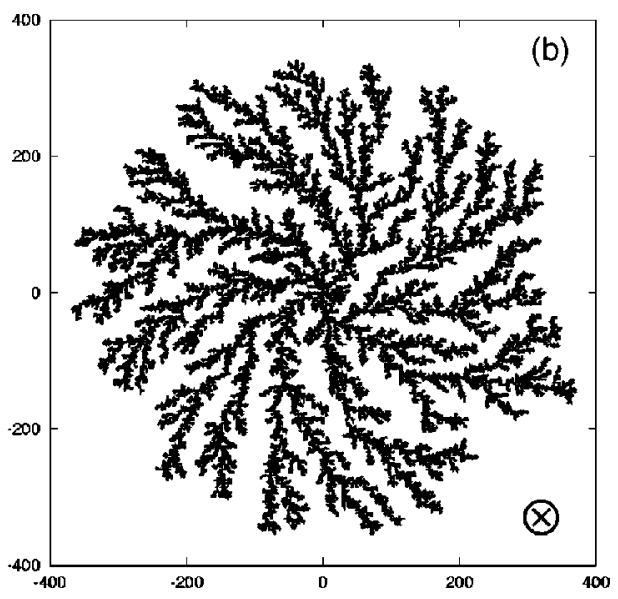

FIG. 8. The first magnetic field model yields spiral patterns but with the wrong chirality for the magnetic field applied (a) vertically upwards to the horizontal cell and (b) vertically downwards. 
fectively just the sum of the forces on individual electrons. The net effect of the Lorentz force is to cause the current to flow in a different direction.

This example differs from the present scenario, however, in that the current is now flowing though a liquid rather than a solid. Each metal ion in the electrolyte is surrounded by a hydration sphere of associated water molecules. Thus the effect of the Lorentz force in changing the path of the ions, which transfer momentum to water molecules beyond the first hydration layer, is to induce a flow of the electrolyte in the cell. The surface of the deposit provides a solid boundary for this flow. The ions in the electrolyte therefore flow past the surface of the deposit.

We thus propose an alternative magnetic field model based not solely on the interaction between the magnetic field and individual ions in solution, but between ions in the Lorentz force-induced flow and the surface of the deposit.

Our solution must therefore be based upon a solution of the convective diffusion equation

$$
\frac{\partial c}{\partial t}=D \nabla^{2} c-\nabla \cdot(c \boldsymbol{v})
$$

where $\boldsymbol{v}$ is the velocity of fluid flow. Taken together with the continuity equation

$$
\boldsymbol{\nabla} \cdot \boldsymbol{v}=0
$$

at equilibrium Eq. (20) reduces to

$$
D\left[\frac{\partial^{2} c}{\partial x^{2}}+\frac{\partial^{2} c}{\partial y^{2}}\right]=v_{x} \frac{\partial c}{\partial x}+v_{y} \frac{\partial c}{\partial y} .
$$

Using the dimensionless variables $\bar{c}=c / c_{o}$, where $c_{o}$ is the bulk concentration; $\bar{x}=x / a$ and $\bar{y}=y / a$, where $a$ is the lattice spacing between adjacent grid points; $\bar{v}_{x}=(a / D) v_{x}$ and $\bar{v}_{y}$ $=(a / D) v_{y}$; we can rewrite Eq. (22) in dimensionless form

$$
\frac{\partial^{2} \bar{c}}{\partial \bar{x}^{2}}+\frac{\partial^{2} \bar{c}}{\partial \bar{y}^{2}}=\bar{v}_{x} \frac{\partial \bar{c}}{\partial \bar{x}}+\bar{v}_{y} \frac{\partial \bar{c}}{\partial \bar{y}} .
$$

As in Sec. II A, casting Eq. (23) in finite terms yields the concentration at grid point $\left(\bar{x}_{i}, \bar{y}_{j}\right)$ relative to the concentrations at its nearest neighbors

$$
\begin{aligned}
\bar{c}\left(\bar{x}_{i}, \bar{y}_{j}\right)= & \bar{c}\left(\bar{x}_{i+1}, \bar{y}_{j}\right)\left[\frac{1}{4}-\frac{\bar{v}_{x}}{8}\right]+\bar{c}\left(\bar{x}_{i-1}, \bar{y}_{j}\right)\left[\frac{1}{4}+\frac{\bar{v}_{x}}{8}\right] \\
& +\bar{c}\left(\bar{x}_{i}, \bar{y}_{j+1}\right)\left[\frac{1}{4}-\frac{\bar{v}_{y}}{8}\right]+\bar{c}\left(\bar{x}_{i}, \bar{y}_{j-1}\right)\left[\frac{1}{4}+\frac{\bar{v}_{y}}{8}\right] .
\end{aligned}
$$

Remembering that angles are defined anticlockwise from the $y$ axis so that $\bar{v}_{x}=-\bar{v} \sin \theta$ and $\bar{v}_{y}=\bar{v} \cos \theta$, we rewrite Eq. (24) as

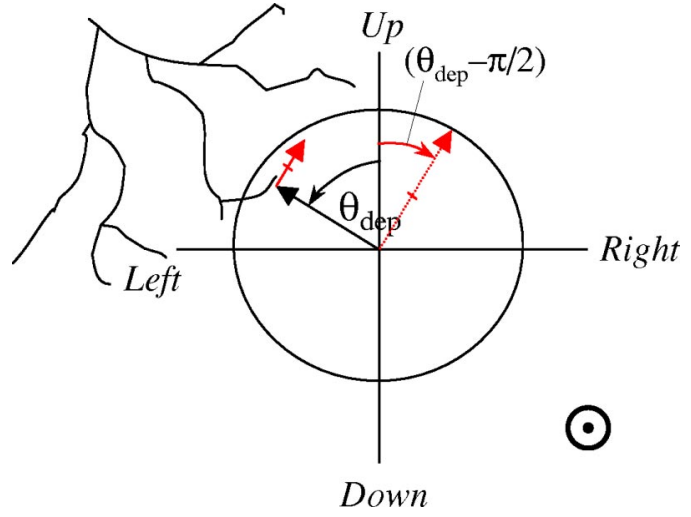

FIG. 9. The nearest occupied site is found by again scanning within a lookup radius $R_{\text {look }}$ about the particle. Flow due to the magnetic field is parallel to the plate, i.e., at an angle $\left(\theta_{\text {dep }} \pm \pi / 2\right)$ depending on the field direction. Here the angle is $\left(\theta_{d e p}-\pi / 2\right)$ modeling a magnetic field applied vertically upwards.

$$
\begin{aligned}
\bar{c}\left(\bar{x}_{i}, \bar{y}_{j}\right)= & \bar{c}\left(\bar{x}_{i+1}, \bar{y}_{j}\right)\left[\frac{1}{4}+\frac{\bar{v}}{8} \sin \theta\right]+\bar{c}\left(\bar{x}_{i-1}, \bar{y}_{j}\right)\left[\frac{1}{4}-\frac{\bar{v}}{8} \sin \theta\right] \\
& +\bar{c}\left(\bar{x}_{i}, \bar{y}_{j+1}\right)\left[\frac{1}{4}-\frac{\bar{v}}{8} \cos \theta\right] \\
& +\bar{c}\left(\bar{x}_{i}, \bar{y}_{j-1}\right)\left[\frac{1}{4}+\frac{\bar{v}}{8} \cos \theta\right] .
\end{aligned}
$$

Note that Eq. (25) has the same form as Eq. (15), with $\bar{v}$ replacing $\bar{E}$.

Now the current density at each point on the surface is perpendicular to the surface at that point, and the Lorentz force acts at right angles to both $\boldsymbol{j}$ and $\boldsymbol{B}$. A flow of electrolyte is therefore established which, close to the deposit, is confined to flow parallel to the solid surface. We therefore introduce the effect of an applied magnetic field by increasing the probability that, close to the deposit, the particle will move perpendicular to the line joining the walker to the nearest point on the surface. We find the nearest point on the surface by scanning within a lookup radius $R_{\text {look }}$ of the particle, as in Sec. II A, and then increase the probability that the particle will move at a tangent to this point (Fig. 9) according to

$$
\begin{gathered}
\mathrm{Up}: \frac{1}{4}+v_{\text {flow }}(B) \cos \left(\theta_{\text {dep }} \pm \pi / 2\right), \\
\text { Down: } \frac{1}{4}-v_{\text {flow }}(B) \cos \left(\theta_{\text {dep }} \pm \pi / 2\right), \\
\text { Left: } \frac{1}{4}+v_{\text {flow }}(B) \sin \left(\theta_{\text {dep }} \pm \pi / 2\right), \\
\text { Right: } \frac{1}{4}-v_{\text {flow }}(B) \sin \left(\theta_{\text {dep }} \pm \pi / 2\right),
\end{gathered}
$$

where $v_{\text {flow }}(B)$ is a multiplicative factor representing the velocity of the flow set up by the magnetic field. [Note that in both magnetic field models, the magnetic field term is added to the electric field terms of Eqs. (16) and (17).] The " \pm " of Eq. (26) is '+' when the magnetic field is applied vertically 

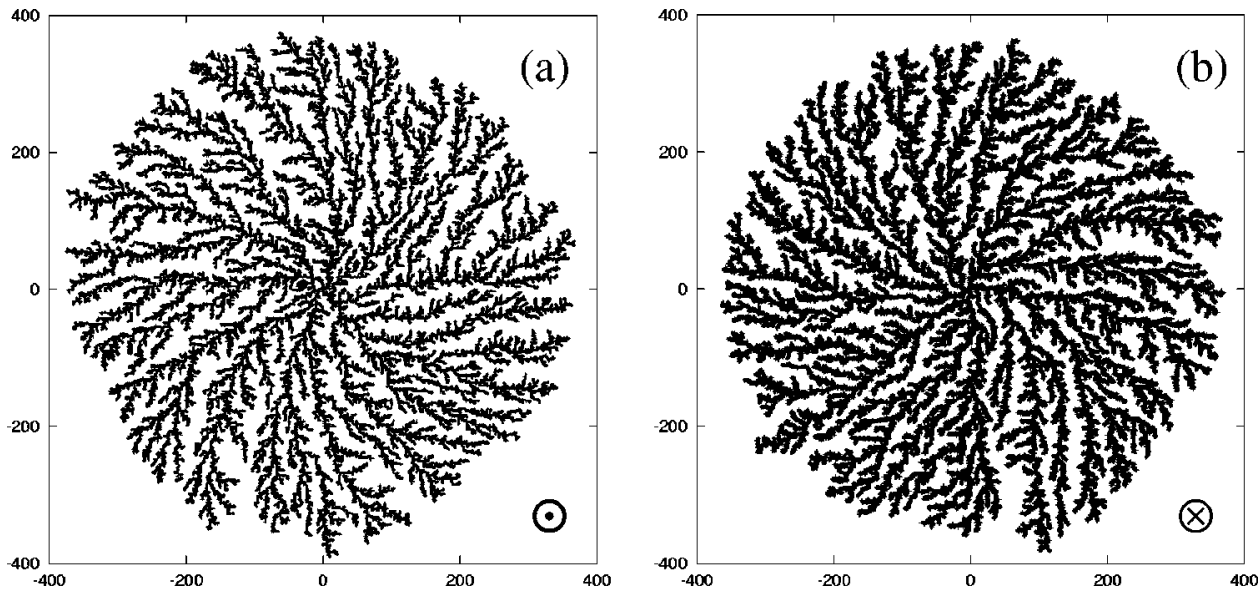

FIG. 10. The improved magnetic field model produces spiral patterns which agree with the experimental result, $v_{\text {flow }}(B)=0.3$ for the magnetic field applied (a) vertically upwards to the horizontal cell and (b) vertically downwards.

downwards to the horizontal cell and "_" when applied vertically upwards.

This model yields chiral results as shown in Fig. 10. In this case, the chirality agrees with the experimental result of Fig. 3.

\section{DISCUSSION}

Both models introduce the magnetic field in terms of the effect of the Lorentz force on moving charges. The first, however, models the effect of the Lorentz force on each individual ion, without consideration for its interaction with the rest of the system. It ignores the fact that (a) the ion is surrounded by water molecules which are associated with that ion by hydrogen bonding; (b) that the magnetic field does not just act on each ion in isolation, but that the net effect of the Lorentz force on the current density towards the deposit is to set up a flow of electrolyte in the cell, and (c) that the surface of the growing deposit marks a boundary for this flow. The flow is parallel to the surface. The role of the surface in convective flow is similarly neglected by Nagatani et al. [25].
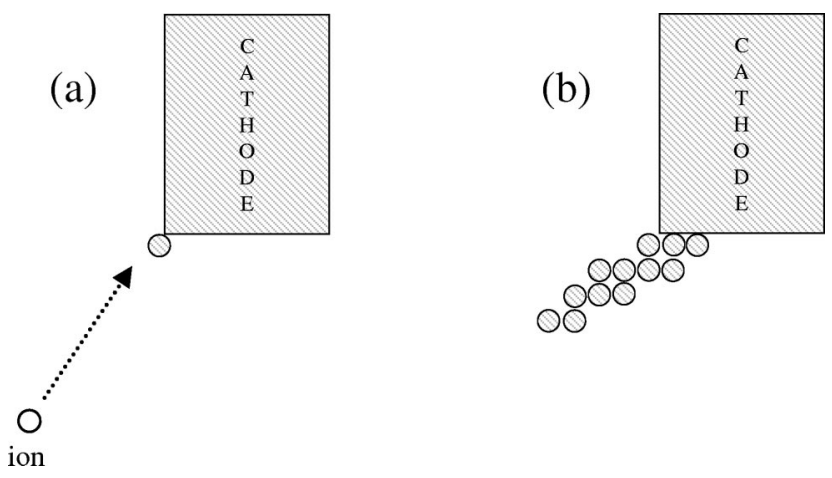

FIG. 11. The incorrect magnetic field model. (a) An applied magnetic field deflects an ion (open circle) in the direction indicated by the dashed arrow. When the ion reaches the surface of the cathode it stops (shaded circle). (b) Over a number of iterations, this results in particles backing-up from existing growth.
An applied magnetic field deflects ions in the same direction in both models. The key failure of the original model, however, is that it neglects to detect in advance the presence of the deposit surface, so that when new particles stick they tend to backup from occupied sites as indicated in Fig. 11. This results in branches which spiral backwards from existing growth. Our new model, on the other hand, recognizes that ions flow along the surface, eventually sticking somewhere on the other side of an existing branch as illustrated in Fig. 12. This is clearly the more accurate model for the experimental system. Recent observations on the chirality of deposits produced in a rotating cell [26,27] confirm this mechanism. A further factor favoring downstream growth may be the concentration depletion effect discussed by Barkey et al. [8].

We must also consider what precisely is being represented by an occupied site in a numerical deposit. A typical experimental electrodeposit contains $\sim 10^{20}$ atoms, while a typical numerical structure consists of $\sim 10^{5}$ occupied sites. These electrodeposits are fractal in nature, and as such are selfsimilar over several orders of magnitude; nonetheless, this discrepancy indicates that an occupied site on the numerical

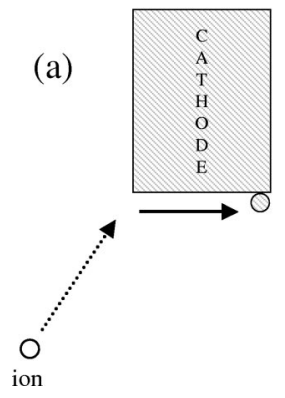

(b)

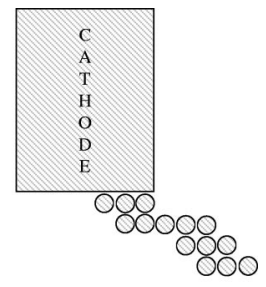

FIG. 12. The new magnetic field model. (a) An applied magnetic field deflects an ion (open circle) in the direction indicated by the dashed arrow. This results in a flow of electrolyte in this direction. The fluid is, however, confined to flow parallel to the solid surface of the electrode as shown by the solid arrow. Ions are swept along the surface until eventually they stick. (b) Over a number of iterations, this results in particles stretching forward from existing growth. 
grid cannot simply represent the reduction of a single metal ion. Recent electron microscope investigations [15] confirm the fractal nature of zinc electrodeposits $\sim 25 \mathrm{~mm}$ in diameter, down to the micron scale. On this scale, however, the fundamental building blocks of the structure are observed to be elementary crystallites $\sim 1 \mu \mathrm{m}$ in length, each containing $\sim 10^{10}$ atoms. A deposit is composed of $\sim 10^{10}$ crystallites.

Given this observation, we consider our model in the context of the work of Fleury et al. [28-30] and Chazalviel [31]. Chazalviel provides a theoretical demonstration that, although the bulk solution remains electrically neutral, a space charge region develops in the immediate vicinity of the cathode. In this anion-poor region (with thickness typically of order a micron at realistic concentrations in the range $10^{-6}-1 \mathrm{M}$ ) an electric field $\boldsymbol{E}^{*}$ develops which is much stronger than that in the bulk. Nucleation of a crystallite by reduction of a cation is most probable in this higher field. A growth phase follows nucleation, and cations continue to be reduced at this point. As the crystallite grows, however, it stretches out into the solution more quickly than the anions can recede. The electric field thus drops, and so too does the likelihood of another nucleation event at that point. The average velocity of growth of the front of the deposit is thus equal to the recession speed of the anions. Fleury shows experimentally that branched electrodeposits develop from such oscillatory nucleation and growth processes. In this context, we consider that an occupied site on our numerical deposit represents not the point of reduction of a single cation, but a point of nucleation and subsequent growth of a micron-sized crystallite.

In Sec. II A we argued that deviations from strict electroneutrality may be modeled as either randomly oriented local fluctuations in the electric field, or a net radial field term which we approximate as an additional external field. In the former case, the fluctuations simply result in a random bias, which is efficiently modeled through the basic random movement of the walker across the lattice. In the latter case, the net internal radial field is absorbed into our term for the externally applied electric field.

We recognize, however, that within the space charge region [31], the electroneutrality approximation breaks down entirely and $z_{c} c_{c} \gg z_{a} c_{a}$, so that Eq. (8)

$$
\nabla^{2} \Phi=-\nabla \cdot E=-\frac{F\left(z_{c} c_{c}-z_{a} c_{a}\right)}{\varepsilon \varepsilon_{o}}=-\rho
$$

relates to a potential $\Phi$ which satisfies the Poisson equation

$$
\nabla^{2} \Phi=-\rho
$$

and Eq. (15) no longer holds; that is, the concentration profile cannot be simplified on a grid in terms of neighboring concentrations and probabilities which sum to one. We assert, however, that an occupied numerical site represents a point on the edge of the typically micron-sized space charge layer where the local field $E^{*}$ is strong enough to initiate nucleation of an individual crystallite. The subsequent growth process as described by Fleury [30] is not considered here.

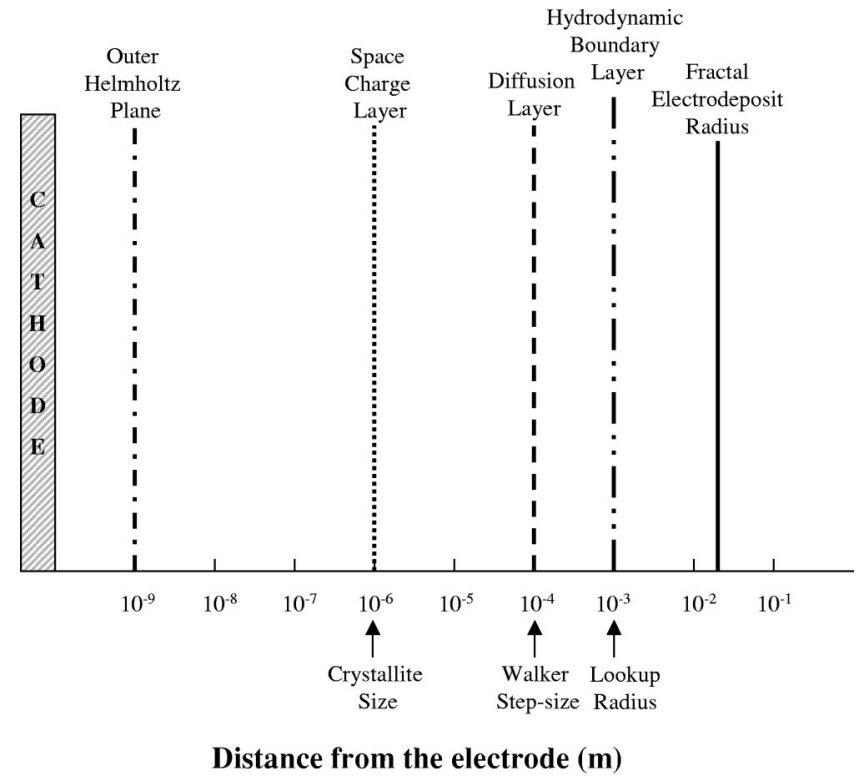

FIG. 13. Length scales in the problem are summarized on a logarithmic scale. Measured from the cathode surface, the Outer Helmholtz Plane is at $\sim 1 \mathrm{~nm}$ [35]; the space charge layer ends at $\sim 1 \mu \mathrm{m}$; the diffusion layer has thickness $\sim 100 \mu \mathrm{m}$; and the hydrodynamic boundary layer has thickness $\sim 1 \mathrm{~mm}$. The latter two are associated with the walker step-size and lookup radius respectively. The length of elementary crystallites in zinc fractal electrodeposits is $\sim 1 \mu \mathrm{m}$.

We also note that it is possible to interpret our result in the context of the electrokinetic effect proposed by Olivier et al. $[32,33]$ to explain the mechanism for the effect of an applied magnetic field on an electrochemical system. In their model, a nonelectrostatic electric field is established parallel to an electrode surface as a result of the interaction between the current density plating to the surface and a perpendicular applied magnetic field. This electric field, by electroosmosis, induces a flow of electrolyte parallel to the surface in the uncompensated solution beyond the outer Helmholtz plane. Olivier et al. contend that this effect alone is sufficient to account for magnetic field effects on electrodeposition. We note, in this context, that the key component of our numerical model is simply the flow of electrolyte parallel to the electrode surface, induced by the magnetic field. Bulk flow of the electrolyte in the cell is not explicit numerically. By considering this flow only in the immediate vicinity of the surface it is possible to reproduce the experimentally determined morphologies. As such, the magnetically activated electrokinetic effect falls under the umbrella of our numerical model as a possible explanation for the mechanism behind the magnetic field effect.

Finally, we return to the question of length scale. The various length scales in the problem are summarized in Fig. 13. What is the physical sense of the step size of the walker? In the final step as the walker attaches to the deposit surface, the step size must relate to the distance over which the concentration of freely diffusing ions passes from $c_{o}$ to zero. This is the thickness of the diffusion layer, over which there is a concentration gradient of the metal cations, which is $\sim 100 \mu \mathrm{m}$ for typical current densities [34] in these cells. An 
$800 \times 800$ grid size therefore corresponds to approximately $8 \times 8 \mathrm{~cm}^{2}$, roughly the dimension of the electrochemical cell, and significantly larger than the size of the deposit. Furthermore, the hydrodynamic boundary layer is typically of order ten times the thickness of the diffusion layer [34] and so is established over approximately ten numerical lattice spacings. In our simulation, magnetic field effects were considered within a lookup radius $R_{\text {look }}$ of ten lattice spacings from the deposit surface. The hydrodynamic boundary layer established in the presence of convective flow, which in this case is induced by an applied magnetic field, is thus associated with the thickness of the lookup radius and lies comfortably within the resolution limit of the simulation.

\section{CONCLUSIONS}

We have adapted the basic diffusion-limited-aggregation model for the growth of fractal electrodeposits to include the effects of finite concentration, migration in an electric field, and magnetic field-induced convection. In each case we show how the adapted DLA model is related to the underlying differential equations. We have corrected an earlier magnetic field model which generates spiral structures, but with a chirality opposite to the experimental observation. The principal failure of this model lies in its neglect of the fact that the ions are in solution in water, and that a force exerted on the ions therefore results in flow of the electrolyte by mo- mentum transfer beyond the hydration sphere immediately associated with each ion. The alternative magnetic field model presented here considers this magnetic field-induced convective flow and the fact that it is confined to pass parallel to the surface of the growing deposit. This model also yields spiral deposits, but this time with the correct chirality.

The DLA model can be adapted to take account of electric and magnetic fields, provided the electrolyte remains approximately charge neutral, and deviations from neutrality can be modeled by electric fields which are either local and randomly oriented or external and radially symmetric. When this is not the case, as within the narrow space charge region near the cathode surface, the Laplace equation for the electric potential must be replaced by a Poisson equation and the DLA approximation breaks down.

We also argue that an occupied site in a numerical deposit does not represent the reduction of a single metal cation. It marks instead the point, at the edge of the space charge layer immediately surrounding the cathode, of nucleation and growth of a micron-sized crystallite containing $\sim 10^{10}$ atoms. The step length of the random walker is identified with the diffusion layer thickness and the lookup radius identified with the hydrodynamic boundary layer thickness.

\section{ACKNOWLEDGMENT}

This work was supported by Science Foundation Ireland as part of the CINSE project.
[1] C. M. de la Condamine, Hist. Mém. Acad. Roy. Sci. 31, 466 (1731).

[2] B. Mandelbrot, The Fractal Geometry of Nature (Freeman, San Francisco, 1977).

[3] T. A. Witten and L. M. Sander, Phys. Rev. Lett. 47, 1400 (1981).

[4] M. Matsushita, M. Sano, Y. Hayakawa, and Y Sawada, Phys. Rev. Lett. 53, 286 (1984).

[5] D. Grier, E. Ben-Jacob, R. Clarke, and L. M. Sander, Phys. Rev. Lett. 56, 1264 (1986).

[6] Y. Sawada, A. Dougherty, and J. P. Gollub, Phys. Rev. Lett. 56, 1260 (1986)

[7] D. G. Grier, D. A. Kessler, and L. M. Sander, Phys. Rev. Lett. 59, 2315 (1987).

[8] D. Barkey, P. Garik, E. Ben-Jacob, B. Miller, and B. Orr, J. Electrochem. Soc. 139, 1044 (1992).

[9] I. Mogi, M. Kamiko, S. Okubo, and G. Kido, Physica B 201, 606 (1994).

[10] I. Mogi and M. Kamiko, J. Cryst. Growth 166, 276 (1996).

[11] I. Mogi, S. Okubo, and Y. Nakagawa, J. Phys. Soc. Jpn. 60, 3200 (1991).

[12] G. Hinds and J. M. D. Coey, J. Appl. Phys. 83, 6447 (1998).

[13] J. M. D. Coey, G. Hinds, C. O’Reilly, and T. R. Ní Mhíocháin, Mater. Sci. Forum 373, 1 (2001).

[14] G. Hinds, F. E. Spada, J. M. D. Coey, T. R. Ní Mhíocháin, and M. E. G. Lyons, J. Phys. Chem. 105, 9487 (2001).

[15] T. R. Ní Mhíocháin, G. Hinds, A. Martin, E. Chang, A. Lai, L.
Costiner, and J. M. D. Coey, Electrochim. Acta (to be published).

[16] Manufactured by Magnetic Solutions Limited, Unit 13, Trinity Enterprise Centre, Pearse St., Dublin 2, Ireland.

[17] H. Mizuseki, K. Tanaka, K. Ohno, and Y. Kawazoe, Modell. Simul. Mater. Sci. Eng. 8, 1 (2000).

[18] H. Mizuseki and Y. Kawazoe, J. Appl. Phys. 87, 4611 (2000).

[19] P. Meakin, Fractals, Scaling and Growth Far from Equilibrium (Cambridge University Press, Cambridge 1998).

[20] John S. Newman, Electrochemical Systems (Prentice-Hall, 1973), Sec. 76.

[21] T. R. Ní Mhíocháin, Ph.D. Thesis, Trinity College, 2001.

[22] J. Erlebacher, P. C. Searson, and K. Sieradzki, Phys. Rev. Lett. 71, 3311 (1993).

[23] R. F. Voss, Phys. Rev. B 30, 334 (1984).

[24] T. R. Ní Mhíocháin, and J. M. D. Coey, Magnetohydrodynamics 37, 169 (2001).

[25] T. Nagatani and F. Saqués, Phys. Rev. A 43, 2970 (1991).

[26] V. Heresanu, R. Ballou, and P. Molho, in Proceedings of the Fifth International Pamir Conference on Fundamental and Applied MHD, edited by A. Alemany, H. Branover, O. Lielausis, and J. P. Chopart (Ramatuelle, France, 2002), Vol. 1.

[27] V. Heresanu, Ph.D. Thesis Laboratoire Louis Néel, 2003.

[28] V. Fleury, J.-N. Chazalviel, and M. Rosso, Phys. Rev. Lett. 68, 2492 (1992).

[29] V. Fleury, J. H. Kaufman, and D. B. Hibbert, Nature (London) 367, 435 (1994). 
[30] V. Fleury, Nature (London) 390, 145 (1997).

[31] J.-N. Chazalviel, Phys. Rev. A 42, 7355 (1990).

[32] A. Olivier, J.-P. Chopart, J. Amblard, E. Merienne, and O. Aaboubi, ACH-Models Chem. 137, 213 (2000).

[33] O. Devos, O. Aaboubi, J.-P. Chopart, E. Merienne, A. Olivier,
C. Gabrielli, and B. Tribollet, Pol. J. Chem. 71, 1160 (1997).

[34] V. G. Levich, Physicochemical Hydrodynamics (Prentice-Hall Eaglewood cliffs, NJ, 1962).

[35] D. B. Hibbert, Introduction to Electrochemistry (Macmillan Physical Science, Basingstoke, 1993). 